\title{
Two Problems in Multiple Access Quantum Communication
}

\author{
Brent J. Yen* and Jeffrey H. Shapiro* \\ ${ }^{*}$ Massachusetts Institute of Technology, Research Laboratory of Electronics, Cambridge, MA 02139, USA
}

\begin{abstract}
Two prior results on the use of quantum resources to increase classical information-transmission capacity are generalized from single-link to multiple-access scenarios. The first is the use of superdense coding over qudit channels. The second is the use of joint measurements over successive uses of the pure-loss Bosonic channel.
\end{abstract}

\section{INTRODUCTION}

Quantum resources may be used to enhance the transmission of classical information. Two prominent single-link examples are as follows: the use of superdense coding [1] to increase capacity by means of prior shared entanglement; and the use of entangling measurements over successive channel uses to increase the capacity achievable over a pureloss Bosonic channel $[2,3]$. In this paper, we shall generalize the preceding results to corresponding instances in multiple access communications, in which two (or more) senders communicate to a common receiver over a shared medium. For the sake of brevity we will limit our presentation to $s=2$ senders; results for $s>2$ appear elsewhere [4].

\section{SUPERDENSE CODING MULTIPLE ACCESS CHANNEL}

Consider a qudit multiple-access channel (MAC) with two senders, Alice and Bob, modeled by the map: $(i, j) \rightarrow \hat{\rho}_{i j}$, in which the output states $\hat{\rho}_{i j}$ live in a finite-dimensional Hilbert space $\mathscr{H}$. The receiver performs a measurement on the state $\hat{\rho}_{i j}$ to learn the input messages $i$ and $j$. Let $p_{i}^{A} p_{j}^{B}$ be a product probability distribution for the letter states $\hat{\rho}_{i j}$, and denote the ensemble-averaged density matrices by

$$
\hat{\rho}=\sum_{i} \sum_{j} p_{i}^{A} p_{j}^{B} \hat{\rho}_{i j}, \quad \hat{\rho}_{i}^{A}=\sum_{j} p_{j}^{B} \hat{\rho}_{i j}, \quad \hat{\rho}_{j}^{B}=\sum_{i} p_{i}^{A} \hat{\rho}_{i j}
$$

and the conditional von Neumann entropies by

$$
H_{A}=\sum_{j} p_{j}^{B} S\left(\hat{\rho}_{j}^{B}\right) \quad \text { and } \quad H_{B}=\sum_{i} p_{i}^{A} S\left(\hat{\rho}_{i}^{A}\right) .
$$

The capacity region for the transmission of classical information over quantum multiple access channels was derived in [5]. It is given by the closure of the convex hull of all $\left(R_{1}, R_{2}\right)$ satisfying

$$
R_{1}<H_{A}-\sum_{i} \sum_{j} p_{i}^{A} p_{j}^{B} S\left(\hat{\rho}_{i j}\right), \quad R_{2}<H_{B}-\sum_{i} \sum_{j} p_{i}^{A} p_{j}^{B} S\left(\hat{\rho}_{i j}\right), \quad R_{1}+R_{2}<S(\hat{\rho})-\sum_{i} \sum_{j} p_{i}^{A} p_{j}^{B} S\left(\hat{\rho}_{i j}\right),
$$

for some product distribution $p_{i}^{A} p_{j}^{B}$.

In a multiple-access superdense coding communication protocol, Alice, Bob, and Charlie share qudits in the initial state $\hat{\rho}_{0}$ that lives in the finite-dimensional Hilbert space $\mathscr{H}_{d_{A}} \otimes \mathscr{H}_{d_{B}} \otimes \mathscr{H}_{d_{C}}$. Alice and Bob apply local unitary operators to encode messages on their qudits, which are then sent to Charlie. Charlie decodes the messages with a measurement on the combined three-qudit system.

Suppose Alice and Bob utilize local unitary operators $\left\{\hat{U}_{i}^{A}\right\}$ and $\left\{\hat{U}_{j}^{B}\right\}$, respectively, with product distribution $p_{i}^{A} p_{j}^{B}$ to encode their messages. By subadditivity, we can give the conditional entropy an upper bound $H_{A}$ as follows,

$$
H_{A} \leq \sum_{j} p_{j}^{B}\left[S\left(\operatorname{tr}_{A}\left(\hat{\rho}_{j}^{B}\right)\right)+S\left(\operatorname{tr}_{B C}\left(\hat{\rho}_{j}^{B}\right)\right)\right] \leq S\left(\hat{\rho}_{B C}\right)+\log d_{A}
$$


where the partial trace of the initial state is $\hat{\rho}_{B C} \equiv \operatorname{tr}_{A}\left(\hat{\rho}_{0}\right)$. Similar bounds are obtained for $H_{B}$ and $S(\hat{\rho})$. Thus, upper bounds on the transmission rates $\left(R_{1}, R_{2}\right)$ for superdense coding with tripartite entanglement are

$$
R_{1} \leq S\left(\hat{\rho}_{B C}\right)-S\left(\hat{\rho}_{0}\right)+\log d_{A}, \quad R_{2} \leq S\left(\hat{\rho}_{A C}\right)-S\left(\hat{\rho}_{0}\right)+\log d_{B}, \quad R_{1}+R_{2} \leq S\left(\hat{\rho}_{C}\right)-S\left(\hat{\rho}_{0}\right)+\log d_{A}+\log d_{B} .
$$

Alice and Bob can achieve these bounds by equiprobable random coding over the Pauli operators in $\mathscr{H}_{d_{k}}$,

$$
\hat{U}_{n m}^{k}=\sum_{l=0}^{d_{k}-1} \exp \left(\frac{2 \pi i l n}{d_{k}}\right)|l\rangle\langle l+m|, \quad n, m=0,1, \ldots, d_{k}-1,
$$

for $k=A, B$, respectively, as shown in [4]. Thus, the capacity of the superdense coding multiple access channel is the set of all rate pairs $\left(R_{1}, R_{2}\right)$ that satisfy the set of inequalities (5).

\section{QUANTUM OPTICAL MULTIPLE ACCESS CHANNEL}

We consider a simple quantum optical multiple access channel in which two senders, Alice and Bob, wish to transmit classical information to a common receiver Charlie, and each sender has access to one input port of a beam splitter with transmissivity $0 \leq \eta \leq 1$. For a single mode of this quantum optical MAC, we have that

$$
\hat{c}=\sqrt{\eta} \hat{a}+\sqrt{1-\eta} \hat{b},
$$

where $\hat{a}$ and $\hat{b}$ are the annihilation operators of Alice's and Bob's input modes, and $\hat{c}$ is the annihilation operator of the mode that Charlie measures.

First, let us suppose the Alice and Bob encode complex-valued input messages $\alpha$ and $\beta$ as coherent states $|\alpha\rangle_{A} \otimes|\beta\rangle_{B}$ with independent input distributions $p_{A}(\alpha)$ and $p_{B}(\beta)$. The corresponding received output state is the coherent state $|\sqrt{\eta} \alpha+\sqrt{1-\eta} \beta\rangle_{C}$, so we will refer to this system as the (single-mode) coherent-state MAC. We assume that the quantum MAC capacity result described by the rate upper bounds (3) is valid for continuous-variable quantum systems. Then, circularly symmetric Gaussian distributions are optimal input distributions, and evaluating the rate upper bounds gives the coherent-state MAC capacity region subject to average photon-number constraints $\bar{n}_{A}$ and $\bar{n}_{B}$ :

$$
R_{1} \leq g\left(\eta \bar{n}_{A}\right), \quad R_{2} \leq g\left((1-\eta) \bar{n}_{B}\right), \quad \text { and } \quad R_{1}+R_{2} \leq g\left(\eta \bar{n}_{A}+(1-\eta) \bar{n}_{B}\right)
$$

where $g(x) \equiv(1+x) \log (1+x)-x \log (x)$. This capacity region, optimized over joint receiver measurements, is larger than what is achievable with the additive Gaussian noise channels associated with homodyne and heterodyne reception [6]

$$
R_{1} \leq \frac{1}{2} \log \left(1+4 \eta \bar{n}_{A}\right), \quad R_{2} \leq \frac{1}{2} \log \left(1+4(1-\eta) \bar{n}_{B}\right), \quad \text { and } \quad R_{1}+R_{2} \leq \frac{1}{2} \log \left(1+4 \eta \bar{n}_{A}+4(1-\eta) \bar{n}_{B}\right),
$$

for homodyne reception and

$$
R_{1} \leq \log \left(1+\eta \bar{n}_{A}\right), \quad R_{2} \leq \log \left(1+(1-\eta) \bar{n}_{B}\right), \quad \text { and } \quad R_{1}+R_{2} \leq \log \left(1+\eta \bar{n}_{A}+(1-\eta) \bar{n}_{B}\right),
$$

for heterodyne reception. Note, however, that the heterodyne-reception capacity region is asymptotically equivalent to the optimum joint-measurement capacity region in the limit $\bar{n}_{A} \rightarrow \infty, \bar{n}_{B} \rightarrow \infty$.

The preceding single-mode results for the coherent-state MAC are easily extended to the case of wideband operation, in which Alice and Bob may employ photons of any frequency, subject to constraints, $P_{A}$ and $P_{B}$, on the average transmitted powers. By means of Lagrange multiplier optimizations we can show that the frequency-dependent average photon numbers- $\bar{n}_{A}(f)$ and $\bar{n}_{B}(f)$-employed by Alice and Bob to realize the capacity regions for optimum, homodyne, and heterodyne reception are all water-filling solutions, and that these capacity regions (in nats/sec) are given by,

$$
R_{1}=\sqrt{\frac{\eta P_{A}}{\pi \hbar}}, \quad R_{2}=\sqrt{\frac{(1-\eta) P_{B}}{\pi \hbar}}, \quad \text { and } \quad R_{1}+R_{2}=\sqrt{\frac{\eta P_{A}+(1-\eta) P_{B}}{\pi \hbar}}
$$

for optimum (joint-measurement) reception and by

$$
R_{1}=\sqrt{\frac{\pi \eta P_{A}}{3 \hbar}}, \quad R_{2}=\sqrt{\frac{\pi(1-\eta) P_{B}}{3 \hbar}}, \quad \text { and } \quad R_{1}+R_{2}=\sqrt{\frac{\pi\left(\eta P_{A}+(1-\eta) P_{B}\right)}{3 \hbar}}
$$


for both homodyne and heterodyne reception. Thus we see that the optimal receiver gives a factor $\pi / \sqrt{3}$ improvement over the conventional—homodyne and heterodyne—-receivers.

Now let us return to the single-mode case and relax our assumption that the transmitters uses coherent-state encoding, i.e., we will allow them to use non-classical states in their quest for the largest possible capacity region. We begin by developing outer bounds on this capacity region. Let Alice and Bob use input states-averaged over their respective random-coding ensembles $-\bar{\rho}_{A}$ and $\bar{\rho}_{B}$ that are subject to the average photon number constraints $\bar{n}_{A}$ and $\bar{n}_{B}$. Because von Neumann entropies are invariant to mean fields, we know that the optimum $\bar{\rho}_{A}$ and $\bar{\rho}_{B}$ will be zero-mean-field states. This, in turn implies, that $\operatorname{tr}\left[\left(\hat{\rho}_{A} \otimes \hat{\rho}_{B}\right) \hat{c}^{\dagger} \hat{c}\right]=\eta \bar{n}_{A}+(1-\eta) \bar{n}_{B}$, from which it is easily shown that

$$
R_{1}+R_{2} \leq S\left(\mathscr{E}\left(\bar{\rho}_{A} \otimes \bar{\rho}_{B}\right)\right) \leq g\left(\eta \bar{n}_{A}+(1-\eta) \bar{n}_{B}\right) .
$$

The sum-rate upper bound in Eq. (13) coincides with the coherent-state MAC result appearing in (8), which is achievable. Hence we have shown that the sum rate for the capacity is achieved by coherent-state encoding in conjunction with optimum (joint-measurement) reception. Moreover, from (10) it can be shown that heterodyne reception is asymptotically optimum for the sum rate in the limit $\eta \bar{n}_{A}+(1-\eta) \bar{n}_{B} \rightarrow \infty$.

To find the upper bounds for the individual rates $R_{1}$ and $R_{2}$, consider a super receiver that has access to both output ports of the beam splitter representing the optical MAC. The individual rate upper bounds reduce to single-user Holevo informations, so upper bounds for the individual rates are $R_{1} \leq g\left(\bar{n}_{A}\right)$ and $R_{2} \leq g\left(\bar{n}_{B}\right)$. We have shown [4] that these rates are asymptotically achievable, in the limit $\bar{n}_{A} \rightarrow \infty$ and $\bar{n}_{B} \rightarrow \infty$, by use of squeezed-state transmitters and homodyne reception.

To develop inner bounds on the capacity region, we apply a capacity result derived in [7] to the quantum optical MAC. Alice and Bob encode their classical messages $\alpha$ and $\beta$ using input states of the form

$$
\hat{\rho}_{A}(\alpha)=\hat{D}(\alpha) \hat{\rho}_{A}(0) \hat{D}^{\dagger}(\alpha) \text { and } \quad \hat{\rho}_{B}(\beta)=\hat{D}(\beta) \hat{\rho}_{B}(0) \hat{D}^{\dagger}(\beta),
$$

where $\hat{\rho}_{A}(0)$ and $\hat{\rho}_{B}(0)$ are zero-mean Gaussian states with variance matrices $V_{A}$ and $V_{B}$, respectively. This is a modulation code for which the coherent-state encoding is the special case in which $\hat{\rho}_{A}(0)$ and $\hat{\rho}_{B}(0)$ are vacuum states. Now, let Alice and Bob have average photon number constraints $\bar{n}_{A}$ and $\bar{n}_{B}$, respectively, and define $V^{\prime}=$ $\eta V_{A}+(1-\eta) V_{B}$. For input photon numbers $\bar{n}_{A}$ and $\bar{n}_{B}$ sufficiently large, the capacity region of the Gaussian MAC is the set of rate pairs that satisfy

$$
\begin{aligned}
R_{1} & \leq g\left(\eta \bar{n}_{A}+(1-\eta)\left(V_{1}^{B}+V_{2}^{B}-1 / 2\right)\right)-g\left(2\left|V^{\prime}\right|^{1 / 2}-1 / 2\right) \\
R_{2} & \leq g\left(\eta\left(V_{1}^{A}+V_{2}^{A}-1 / 2\right)+(1-\eta) \bar{n}_{B}\right)-g\left(2\left|V^{\prime}\right|^{1 / 2}-1 / 2\right) \\
R_{1}+R_{2} & \leq g\left(\eta \bar{n}_{A}+(1-\eta) \bar{n}_{B}\right)-g\left(2\left|V^{\prime}\right|^{1 / 2}-1 / 2\right) .
\end{aligned}
$$

This achievable rate region reduces to the coherent-state formulas, Eq. (8), when $V_{A}=V_{B}=I / 4$. Furthermore, the Gaussian-state encoding is a sum-rate achieving code, i.e., (17) coincides with (13) whenever $\mathscr{E}\left(\hat{\rho}_{A}(0) \times \hat{\rho}_{B}(0)\right)$ is a pure state. Although non-classical light is not necessary to achieve the sum-rate capacity, this is not the case for the individual rates. Indeed, it is possible to find $V_{A}$ and $V_{B}$, for example,

$$
V_{A}=\left(\begin{array}{cc}
\frac{1}{32} & 0 \\
0 & 2
\end{array}\right) \quad \text { and } \quad V_{B}=\left(\begin{array}{cc}
\frac{1}{32} & 0 \\
0 & 2
\end{array}\right)
$$

such that the Gaussian-state region is larger than the coherent-state region (8). Numerical optimization can further enlarge the capacity region beyond that achieved by this example, but we do not know how to determine the capacity region achieved by Gaussian codes analytically. Our quantum optical MAC results are illustrated in Fig. 1. Here we have plotted the sum rate for a single-mode quantum optical MAC with $\eta=1 / 2, \bar{n}_{A}=10$ and $\bar{n}_{B}=8$, along with the capacity region for heterodyne reception, the individual rate limits for coherent-state encoding, and the individual rate limits for the Gaussian-state encoding from Eq. (18).

\section{CONCLUSION}

We treated two problems in multiple access quantum communication. First, we considered the superdense coding protocol for transmitting classical information over a quantum multiple access channel belonging to a finite-dimensional 


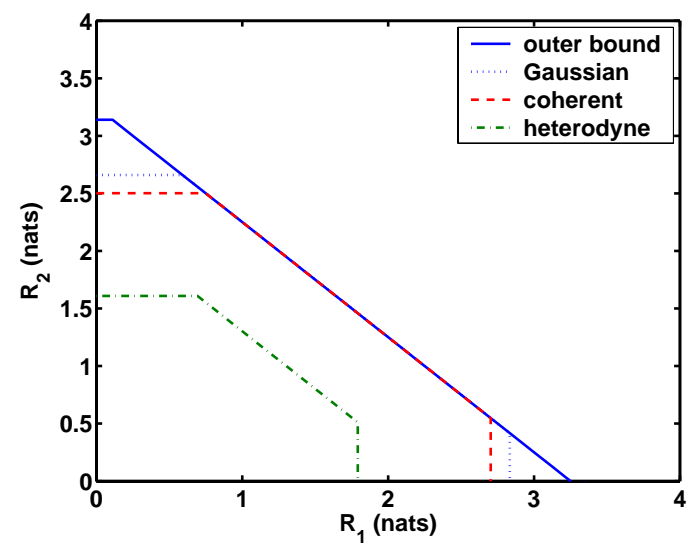

FIGURE 1. Inner and outer bounds on the capacity region for the single-mode quantum optical MAC with $\eta=1 / 2, \bar{n}_{A}=10$, and $\bar{n}_{B}=8$ The outer bound constrains this capacity region to be below the solid line. The capacity region for coherent-state encoding with heterodyne reception lies below the dash-dot line. The capacity region for coherent-state encoding and optimum (joint-measurement) reception lies below both the dashed and the solid lines, i.e., the sum-rate limit is achieved by coherent-state encoding. An inner bound on the capacity region lies below both the dotted and solid lines. The individual-user portions of this inner bound result from Gaussian-state encoding with input variance matrices $V_{A}$ and $V_{B}$ given by (18).

Hilbert space. There we showed that the capacity region of the three-party superdense coding channel is defined by the set of rates satisfying the bounds in (5). Second, we studied the capacity of the quantum optical MAC. For coherentstate encoding-i.e., for classical-light source transmitters-we derived the capacity regions for both single-mode and wideband operation, and compared them with corresponding results when either homodyne or heterodyne reception is employed in lieu of the optimal (joint-measurement) receiver. Furthermore, we derived an outer bound on the sum rate that coincides with what is achievable with coherent-state encoding. We also exhibited outer bounds on the individual rates that are achieved, in the limit of $\bar{n}_{A} \rightarrow \infty$ and $\bar{n}_{B} \rightarrow \infty$, by squeezed-state encoding and homodyne reception. Finally, we used Gaussian-state encoding to develop inner bounds on the individual rates that are tighter than the coherent-state results.

\section{ACKNOWLEDGMENTS}

This work was supported by the DoD Multidisciplinary University Research Initiative (MURI) program administered by the Army Research Office under Grant DAAD19-00-0177.

\section{REFERENCES}

1. C. H. Bennett and S. J. Wiesner, Phys. Rev. Lett., 69, 2881, (1992).

2. V. Giovannetti, S. Guha, S. Lloyd, L. Maccone, J. H. Shapiro, and H. P. Yuen, Phys. Rev. Lett., 92, 027902 (2004).

3. J. H. Shapiro, V. Giovannetti, S. Guha, S. Lloyd, L. Maccone, and B. J. Yen, "Capacity of Bosonic communications," in S. M. Barnett, ed., Proceedings of the Seventh International Conference on Quantum Communication, Measurement and Computing (American Institute of Physics, New York, 2004).

4. B. J. Yen, "Multiple-user quantum optical communication," Ph.D. thesis, MIT, 2004.

5. A. Winter, IEEE Trans. Inform. Theory, 47, 3059 (2001).

6. T. M. Cover and J. A. Thomas, Elements of Information Theory (Wiley, New York, 1991).

7. A. S. Holevo and M. Sohma and O. Hirota, Phys. Rev. A, 59, 1820 (1999). 